\title{
Relaxation and curvature-induced molecular flows within multicomponent membranes
}

\author{
Richard G. Morris* \\ Theoretical Physics, The University of Warwick, Coventry CV4 7AL, United Kingdom \\ (Received 8 October 2013; revised manuscript received 18 February 2014; published 9 June 2014)
}

\begin{abstract}
The quantitative understanding of membranes is still rooted in work performed in the 1970s by Helfrich and others, concerning amphiphilic bilayers. However, most biological membranes contain a wide variety of nonamphiphilic molecules too. Drawing analogy with the physics of nematic-non-nematic mixtures, we present a dynamical (out-of-equilibrium) description of such multicomponent membranes. The approach combines nematohydrodynamics in the linear regime and a proper use of (differential-) geometry. The main result is to demonstrate that one can obtain equations describing a cross-diffusion effect (similar to the Soret and Dufour effects) between curvature and the (in-membrane) flow of amphiphilic molecules relative to nonamphiphilic ones. Surprisingly, the shape of a membrane relaxes according to a simple heat equation in the mean curvature, a process that is accompanied by a simultaneous boost to the diffusion of amphiphiles away from regions of high curvature. The model also predicts the inverse process, by which the forced bending of a membrane induces a flow of amphiphilic molecules towards areas of high curvature. In principle, numerical values for the relevant diffusion coefficients should be verifiable by experiment.
\end{abstract}

DOI: 10.1103/PhysRevE.89.062704

PACS number(s): 87.16.D-, 82.20.-w, 05.70.Ln

\section{INTRODUCTION}

The behavior of the cell membrane is crucially important to a wide variety of processes in biology [1]. However, since the underlying construction of almost all biological membranes is that of an amphiphilic bilayer, much of the physics literature has so far focused on understanding simple bilayers and their closed-form counterparts, vesicles [2,3]. Despite intensive research over the last 50 years, such approaches have failed to properly describe the role of the biological membranes in commonly observed phenomena, such as cell locomotion, for example. The point is that often the nonamphiphilic component of the membrane is important (in the case of cell locomotion, consider so-called membrane-to-cortex attachment proteins embedded in the bilayer). In this paper we attempt a consistent dynamical treatment of such multicomponent membranes, which makes quantitative predictions about the dynamical relationship between curvature and the diffusion of amphiphiles relative to nonamphiphiles. The hope is that such ideas might act as a base onto which successful predictive models of the above phenomena can be built, and more.

The modern quantitative description of bilayers was pioneered by Helfrich [4] in the 1970s (and independently by Canham [5] and Evans [6]) and has not advanced greatly since. Drawing parallels between the amphiphiles of a bilayer and the rodlike molecules of nematic liquid crystals, Helfrich adapted expressions for the Frank free energy [7] by replacing the director with the normal to the membrane surface. The result was a local free energy per unit area of the form

$$
\frac{\kappa}{2}\left(2 H-C_{0}\right)^{2}+\kappa_{g} K \text {. }
$$

In the normal way, $H$ and $K$ are just the mean and Gaussian curvatures, respectively [8], and $C_{0}$-the spontaneous curvature-is a constant. The quantities $\kappa$ and $\kappa_{g}$ are called the bending rigidity and elastic modulus of Gaussian curvature, respectively, and are also constant.

\footnotetext{
*r.g.morris@warwick.ac.uk
}

Strictly, Eq. (1) describes the free energy of a monolayer rather than a bilayer, and therefore a number of attempts at improvement have been made (e.g., [9,10]). However, due to the similarity between monolayers with different head-tail interactions and bilayers with differing leaf densities, both the original model and its relatives have been used extensively in the physics literature [3], most notably in the static description of vesicles under constraint [11-17]. Only recently have attempts been made to write nonequilibrium descriptions of membrane dynamics, but so far the focus has been solely on vesicles [18-22]. Taking inspiration from such studies, this article goes back to the original model of Helfrich and demonstrates that it can be extended by analogy with nematohydrodynamics in the linear regime. As we show, such an approach permits the incorporation of additional nonamphiphilic components and leads to a membrane description in terms of both curvature and in-membrane molecular flows.

\section{FREE ENERGY OF THE MEMBRANE}

The starting point is to make two important observations. First, biological membranes are made not only from amphiphiles, but from a whole host of other nonamphiphilic molecules (cholesterol, carbohydrates, proteins, protein channels, fat-soluble molecules, etc.). Second, in most biological systems the temperature is sufficiently high that the molecules in the membrane effectively form a two-dimensional fluid which, when close to equilibrium, can be described by linear nonequilibrium thermodynamics (LNET) [23]. In this framework, the geometry of the problem then enters through the Gibbs free energy, so it is important to ensure a consistent thermodynamic formulation. Recall then that LNET assumes a local equilibrium, such that the Gibbs relation,

$$
T d s=d u+p d v-\{d g\}_{T, p},
$$

is obeyed at every point in space and time. Here $T$ is temperature, $p$ is pressure, and, following the literature, we use $s$, the specific entropy, given by $S / M$, where $S$ is the usual entropy and $M$ is mass. Similarly, $u=U / M$ is specific 
internal energy, $v=V / M$ is the specific volume, and $g=u-$ $T s+p v$ is the specific Gibbs energy. For clarity, subscripted brackets, $\{\cdots\}_{T, p}$, are used to indicate that both temperature and pressure are held constant. We imagine a membrane as a two-dimensional fluid only one molecule thick. The fluid is an incompressible mixture of rodlike amphiphiles (henceforth referred to simply as lipids) and pointlike "other" molecules. The other molecules are pointlike in the sense that they have no orientation, or, more specifically, we associate with them no energy contribution that is a function of the membrane shape. (They are still assumed to have mass and to occupy volume in the normal way). For simplicity, we take a highly idealized approach and assume that all such pointlike molecules are thermodynamically equivalent (though it should be noted that a full multicomponent treatment is still possible, if tedious). In this simplified case, the specific Gibbs energy is just

$$
g=\sum_{k} \mu_{k} c_{k}+g^{\mathrm{nem}},
$$

where, in the first term, the concentration and chemical potential of component $k$ are given by $c_{k}=M_{k} / M$ and $\mu_{k}$, respectively, with $k \in\{l, o\}$ ( $l$ for "lipids" and $o$ for "other"). If the nonamphiphilic molecules were known, then the subscript would simply label them, in turn, $k \in\{l, 1,2,3, \ldots$, etc. $\}$. The second term in (3) is the energy associated with the nematic nature of the lipid molecules, which can be approximated by an elastic description $[9,24]$ of the chemical interactions between the head groups and between the tails groups of the lipid molecules. The rest of this section briefly reviews such a theory (and its assumptions) in order to highlight certain important caveats that arise due to our description being both dynamical and multicomponent.

\section{A. Elastic description of amphiphilic interactions}

The benefits of an elastic theory are twofold. First, the system can be written formally in terms of a single well-defined "neutral-torque" surface $\mathcal{S}$, using a standard procedure. Second, it provides a description for the quantities $\kappa, \kappa_{g}$, and $C_{0}$ in terms of geometrical variables, allowing the assumptions of this approach to be made plain. The standard route is to write $g^{\text {nem }}$ as a sum of two quadratic terms,

$$
g^{\text {nem }}=k_{\mathrm{h}}\left(a_{\mathrm{h}}-a_{\mathrm{h}, 0}\right)^{2}+k_{\mathrm{t}}\left(a_{\mathrm{t}}-a_{\mathrm{t}, 0}\right)^{2},
$$

where subscripts are used to indicate either head or tail groups. For example, $a_{\mathrm{h}}=A_{\mathrm{h}} / M$ is the area per unit mass on a surface defined to intersect the head groups of all the molecules, while $a_{\mathrm{t}}=A_{\mathrm{t}} / M$ is defined in a similar way for the molecular tails. Both $k_{\mathrm{h}}$ and $k_{\mathrm{t}}$ are constants, as are the quantities $a_{\mathrm{h}, 0}$ and $a_{\mathrm{t}, 0}$, defined as the area per unit mass at equilibrium for head and tail groups, respectively. The projection onto a neutral-torque surface is described in $[24,25]$ and recapitulated (with some small modifications) in Appendix A. The result is that, apart from a term which describes the free energy associated with a lateral tension (which for our model is assumed constant), Eq. (1) is recovered. However, contrary to Helfrich's approach, the values of $\kappa, \kappa_{g}$, and $C_{0}$ are no longer constant, and depend on $a$, the local area per mass [see Eqs. (A10) to (A13)]. This conflict has previously been resolved by the authors of [10] by minimizing the free energy under the constraint of a fixed number of particles in the monolayer (the result is that $a$ is constant up to $\mathcal{O}\left(\delta^{2}\right)$, where $\delta$ is the length along the long axis of the lipid molecules). However, since our description includes the behavior of nonamphiphilic molecules, this result can only be applied as a lowest order approximation when the concentration of nonamphiphiles is low. It is therefore important to state that, physically, we assume to always be in the regime of constant bending rigidity, when the nonamphiphilic molecules do not materially affect $\kappa$ and only contribute to free energy through the first term of (3).

\section{B. Gaussian curvature}

It is also necessary to remark on the Gaussian curvature $K$. While $K$ is routinely ignored in equilibrium studies of vesicles [3] due to the Gauss-Bonnet theorem, such an approach cannot be applied here as it relies on the integration of the membrane energy over a closed surface. However, progress can be made by closely following nematohydrodynamics, where a standard assumption is that the dominant contributions to the free energy of a nematic fluid come from terms proportional to the director and its first spatial derivatives [26,27]. In our case, since we may identify the director with the normal $n$ of the surface $\mathcal{S}$, this amounts to ignoring all terms in (1) that contain higher spatial derivatives of $\boldsymbol{n}$. Leaving the details to Appendix B (geometry will also be discussed in the next section) the important point is that, alongside the standard formula $H=\boldsymbol{\nabla} \cdot \boldsymbol{n} / 2$, the Gaussian curvature can be shown to be given by

$$
K=\frac{1}{2}\left[(2 H)^{2}+\boldsymbol{n} \cdot\left(\nabla^{2} \boldsymbol{n}\right)\right],
$$

which involves second spatial derivatives of $\boldsymbol{n}$. As a result, fully incorporating the effects of the Gaussian curvature is left for further work, and in this treatment we retain only the first term of (5). Taking the above into account, the lipid interactions only contribute to the Gibbs energy via the mean curvature:

$$
g^{\mathrm{nem}}=\frac{\kappa}{2}\left(2 H-C_{0}\right)^{2}+\frac{\kappa_{g}}{2}(2 H)^{2} .
$$

For the purposes of thermodynamics, it is helpful to identify extensive and intensive contributions in Eq. (6). Therefore, noting from (A13) that $\kappa_{g}$ is linear in $\kappa$, and in order to conform with the conventions of nematohydrodynamics, we define the intensive variable $\kappa^{\prime}=\kappa / \rho$, and the corresponding extensive variable $\psi=\frac{\rho}{2}\left(2 H-C_{0}\right)^{2}+\frac{\rho \kappa_{g}}{2 \kappa}(2 H)^{2}$, where $\rho=M / V$ is the usual mass density.

From here, it follows that

$$
g^{\text {nem }}=\kappa^{\prime} \psi, \quad \text { and } \quad\{d g\}_{T, p, c_{i}}=\kappa^{\prime} d \psi,
$$

which, when returning to the local equilibrium condition, gives

$$
T d s=d u+p d v-\sum_{k} \mu_{k} d c_{k}-\kappa^{\prime} d \psi .
$$

\section{LNET FOR (CURVED) MEMBRANES}

The usual LNET approach is to manipulate the time derivative of (7) through careful application of conservation laws and constitutive relations and then to compare the result 
with the equation for local entropy balance,

$$
\rho \frac{d s}{d t}=-\nabla \cdot \boldsymbol{J}_{\mathrm{s}}+\sigma .
$$

(Here $\boldsymbol{J}_{\mathrm{s}}$ is the convective entropy flux, and $\sigma$ is the entropy production term.) Under normal circumstances, this tactic then identifies the source term with a bilinear sum of the thermodynamic forces and fluxes, from which Onsager relations can be deduced.

Before proceeding, however, recall that these relations are now defined on the $2 \mathrm{D}$ surface $\mathcal{S}$, which is assumed to be regular and parametrized by variables $u$ and $v$. Any point on the surface is then defined by the position vector $\boldsymbol{r}=\boldsymbol{r}(u, v)$, and the tangent space at each point is spanned by the two vectors $\boldsymbol{r}_{\alpha} \equiv \partial \boldsymbol{r} / \partial q^{\alpha}$. Here $\alpha, \beta \in\{1,2\}$-and similarly for all Greek indices-where $q^{1}=u$ and $q^{2}=v$. Using the shorthand notation $\partial_{\alpha} \equiv \partial / \partial q^{\alpha}$, the gradient operator becomes

$$
\nabla \equiv g^{\alpha \beta} \boldsymbol{r}_{\beta} \partial_{\alpha}
$$

where $g_{\alpha \beta}=\boldsymbol{r}_{\alpha} \cdot \boldsymbol{r}_{\beta}$ is the metric tensor and $g^{\alpha \beta}$ its inverse. For membranes with a finite thickness, one must typically compute corrections to diffuse processes that are caused by curvature [28]. However, since the monolayer is assumed to be only one molecule thick, these corrections can be ignored and most of the traditional conservation laws (e.g., mass and internal energy) carry over without any modifications. For a system at constant uniform temperature, the application of these laws (Appendix C) gives

$$
\rho T \frac{d s}{d t}=-\Pi:(\nabla \boldsymbol{v})^{\top}+\sum_{k} \mu_{k} \nabla \cdot \boldsymbol{J}_{k}-\rho \kappa^{\prime} \frac{d \psi}{d t},
$$

where $\boldsymbol{J}_{k}=\rho_{k}\left(\boldsymbol{v}_{k}-\boldsymbol{v}\right)$ is the local diffusion flow, and $\Pi=$ $\mathrm{P}-p \mathrm{l}$ is the nonhydrostatic part of the pressure tensor. (Here $\rho_{k} \equiv M_{k} / V$ are partial mass densities, and $\boldsymbol{v} \equiv \sum_{k} \boldsymbol{v}_{k} \rho_{k} / \rho$ defines both the barycentric velocity $v$ and the partial velocities $\boldsymbol{v}_{k}$.) The notation uses sans-serif font for a (rank 2) tensor, a superscript $T$ to denote the transpose, and a colon to represent the trace of an interior product; i.e., the term $\Pi:(\nabla v)^{\top}$ is written in component form as $\Pi_{i j} g^{\alpha \beta}\left(r_{\alpha}\right)_{i} \partial_{\beta} v_{j}$, where a sum is implicit for repeated indices, both Greek and Latin. Here Latin indices are the usual Cartesian components in three dimensions such that $\left(r_{\alpha}\right)_{i}$ is the $i$ th component of the tangent vector $\boldsymbol{r}_{\alpha}$.

As previously mentioned, the traditional liquid crystals approach for computing the time-derivative of free energies with nematic order-such as the final term in (11) - is to assume a dependence on $\boldsymbol{n}$ and its first spatial derivatives $\nabla \boldsymbol{n}$ and then impose straightforward constitutive relations for these quantities [26,27]. For our case, since $\psi$ was constructed with these constraints in mind, the final term in (11) can be computed directly from $H=\nabla \cdot \boldsymbol{n} / 2$, although as we show, care must be taken when differentiating the gradient operator. Specifically, by recalling the definition of $\psi$, the time derivative can be written as

$$
\frac{d \psi}{d t}=\frac{\partial \psi}{\partial \rho} \frac{d \rho}{d t}+\frac{\partial \psi}{\partial(\nabla \cdot \boldsymbol{n})} \frac{d(\nabla \cdot \boldsymbol{n})}{d t} .
$$

Here if the gradient that appears in the last term was just the usual operator (and not restricted to the surface), then

$$
\frac{d}{d t}(\nabla \cdot \boldsymbol{n})=\nabla \cdot \frac{d \boldsymbol{n}}{d t},
$$

and equations similar to those that appear in the theory of nematic liquid crystals could be recovered $[26,27]$. In turn, this leads to terms in (11) that are either convective or bilinear in forces and fluxes, which is required by LNET and therefore desirable. With this in mind, the part that is of interest in (12) is the proper time derivative of $\boldsymbol{\nabla} \cdot \boldsymbol{n}$, which in component form becomes

$$
\frac{d}{d t}\left[g^{\alpha \beta}\left(r_{\alpha}\right)_{i} \partial_{\beta} n_{j}\right]=\frac{d}{d t}\left[g^{\alpha \beta}\left(r_{\alpha}\right)_{i}\right] \partial_{\beta} n_{j}+g^{\alpha \beta}\left(r_{\alpha}\right)_{i} \frac{d}{d t}\left[\partial_{\beta} n_{j}\right] .
$$

At this stage we notice that the director $\boldsymbol{n}$ is an average over local orientations of the amphiphilic molecules only; therefore, we may use the material derivative

$$
\frac{d}{d t}=\frac{\partial}{\partial t}+\boldsymbol{v}_{l} \cdot \nabla
$$

where the velocity is that of the lipids (amphiphiles). Applying (15), the last term in (14) can be expanded (again, in component form) to give

$$
\begin{aligned}
& g^{\alpha \beta}\left(r_{\alpha}\right)_{i} \frac{d}{d t}\left[\partial_{\beta} n_{j}\right] \\
& \quad=g^{\alpha \beta}\left(r_{\alpha}\right)_{i} \partial_{\beta}\left(\frac{\partial n_{j}}{\partial t}\right)+g^{\alpha \beta}\left(r_{\alpha}\right)_{i} g^{\mu \nu}\left(v_{l}\right)_{k}\left(r_{\mu}\right)_{k} \partial_{\nu} \partial_{\beta} n_{j} .
\end{aligned}
$$

Manipulating derivatives, one can then show that the second term of (16) is just equivalent to

$$
g^{\alpha \beta}\left(r_{\alpha}\right)_{i} \partial_{\beta}\left[g^{\mu \nu}\left(v_{l}\right)_{k}\left(r_{\mu}\right)_{k}\right] \partial_{\nu} n_{j},
$$

which, when combined with (14) makes it clear that the condition (13) is only satisfied if

$$
\frac{d}{d t}\left(g^{\alpha \beta} \boldsymbol{r}_{\alpha}\right)=\nabla\left[g^{\alpha \beta}\left(\boldsymbol{v}_{l} \cdot \boldsymbol{r}_{\alpha}\right)\right] .
$$

The simplest way of understanding the ramifications of this relation is to note that it causes the distortion stress to vanish (apart from a straightforward density-dependent term). That is, it is implicitly assumed that there is no energy cost (and therefore no force per unit area) arising as a result of deformations which move the relative positions of the molecules but keep the director field fixed. Mathematically, this is certainly self-consistent with a free energy that is only based on the relative orientation of the molecules, viz. Eq. (4). Physically, since both the lateral tension and the bending rigidity are constant and uniform, any membrane configuration that leads to the same director field must be thermodynamically equivalent.

The main corollary of (18) is that it implies

$\rho T \frac{d s}{d t}=-\nabla \cdot \boldsymbol{J}^{\mathrm{nem}}-\Pi^{\prime}:(\nabla \boldsymbol{v})^{\top}+\sum_{k} \mu_{k} \boldsymbol{\nabla} \cdot \boldsymbol{J}_{k}-\boldsymbol{h} \cdot \frac{d \boldsymbol{n}}{d t}$,

where

$$
\boldsymbol{h}=-\kappa^{\prime} \nabla\left[\rho \frac{\partial \psi}{\partial(\nabla \cdot \boldsymbol{n})}\right]=-2 \rho \bar{\kappa} \nabla H
$$

is the molecular field (constant density), $\Pi^{\prime}=\Pi-$ $\kappa^{\prime} \mid \rho^{2}(\partial \psi / \partial \rho)$ is the viscous stress, and $\boldsymbol{J}^{\mathrm{nem}}$ is a convective "nematic flux" term. [We have introduced the shorthand 
$\bar{\kappa}=\left(\kappa+\kappa_{g}\right) / \rho$, for simplicity]. Before making the comparison with (9), however, a number of constraints can be implemented to make the analysis simpler. First, it is standard to separate out the temperature dependence by manipulating derivatives. Second, we must recognize that the barycentric diffusion flows are not independent; i.e., $\boldsymbol{J}_{l}+\boldsymbol{J}_{o}=0$. Finally, since we stipulate that all molecules point normal to the surface, contributions from the antisymmetric part of $\Pi$ can be neglected (this arises from conservation of angular momentum; see [23,29] and Appendix D). With these modifications, one may identify the entropy production term as

$$
\begin{aligned}
\sigma= & -\frac{1}{T} \Pi^{\mathrm{s}}:(\nabla \boldsymbol{v})^{\mathrm{s}}-\frac{1}{T} \boldsymbol{J}_{l} \cdot\left\{\nabla\left(\mu_{l}-\mu_{o}\right)\right\}_{T, p, \kappa^{\prime}} \\
& -\frac{1}{T} \boldsymbol{h} \cdot \frac{d \boldsymbol{n}}{d t},
\end{aligned}
$$

where a superscript $s$ is used to indicate the symmetric part. It is this form that will lead to reciprocal relations of the Onsager type.

\section{RECIPROCAL LINEAR RELATIONS AND CROSS-DIFFUSION}

Invoking the Cure principle and only writing linear relations for forces and fluxes of the same tensorial character, we focus on the coupled vector relations,

$$
\begin{aligned}
\boldsymbol{J}_{l} & =-L_{\mathrm{dd}} \frac{\left\{\nabla\left(\mu_{l}-\mu_{o}\right)\right\}_{T, p, \kappa^{\prime}}}{T}-L_{\mathrm{dn}} \frac{\boldsymbol{h}}{T}, \\
\frac{d \boldsymbol{n}}{d t} & =-L_{\mathrm{nd}} \frac{\left\{\nabla\left(\mu_{l}-\mu_{o}\right)\right\}_{T, p, \kappa^{\prime}}}{T}-L_{\mathrm{nn}} \frac{\boldsymbol{h}}{T},
\end{aligned}
$$

where the Onsager coefficients $L_{\mathrm{dd}}, L_{\mathrm{nn}}$, and $L_{\mathrm{nd}}=L_{\mathrm{dn}}$ are labeled by subscripts " $d$ " for diffusion and " $n$ " for nematic. It is these linear relations that couple lipid diffusion to curvature and which contain the main result of this paper. However, the result is best cast in terms of a cross-diffusion effect such as the Soret or Dufour effects [23]. To see this, we use the Gibbs-Duhem relation for our system

$$
c_{l}\left\{\nabla \mu_{l}\right\}_{T, p, \kappa^{\prime}}+c_{o}\left\{\nabla \mu_{o}\right\}_{T, p, \kappa^{\prime}}=0 .
$$

From here, using the fact the $c_{o}+c_{l}=1$, it is clear that

$$
\left\{\nabla\left(\mu_{l}-\mu_{o}\right)\right\}_{T, p, \kappa^{\prime}}=\frac{\mu_{l l}^{c}}{c_{o}} \nabla c_{l},
$$

where the shorthand notation of [23] has been used: $\mu_{l l}^{c} \equiv\left(\partial \mu_{l} / \partial c_{l}\right)_{T, p, \kappa^{\prime}}$. On physical grounds, we expect that $\left.\mu_{l l}^{c}\right|_{c_{1}=0}=0$, that is, the rate of change of the lipid-chemical potential with respect to the concentration of lipids is zero when the system can no longer accommodate any more lipids. We may also reasonably expect that $\mu_{l l}^{c}$ is a monotonically decreasing function of $c_{l}$. With these constraints in mind, we make the simplest assumption possible: that $\mu_{l l}^{c}=A_{l l}^{c}(1-$ $\left.c_{l}\right)=A_{l l}^{c} c_{o}$, where $A_{l l}^{c}=$ constant. Substituting this result, along with (20), into the linear relations (22) and then taking the divergence on both sides of the resulting expressions gives

$$
\begin{aligned}
\frac{d c_{l}}{d t} & =D_{\mathrm{dd}} \nabla^{2} c_{l}-2 D_{\mathrm{dn}} \nabla^{2} H, \\
\frac{d H}{d t} & =-\frac{A_{l l}^{c}}{2 \bar{\kappa}} D_{\mathrm{nd}} \nabla^{2} c_{l}+D_{\mathrm{nn}} \nabla^{2} H,
\end{aligned}
$$

where both (13) and the condition for conservation of mass, $\rho\left(d c_{l} / d t\right)=-\nabla \cdot J_{l}$, have been used, and $\nabla^{2}$ is the Laplace-Beltrami operator (Laplacian on the surface). The new coefficients comprise two "direct" terms-a diffusion coefficient,

$$
D_{\mathrm{dd}}=\frac{L_{\mathrm{dd}} A_{l l}^{c}}{\rho T},
$$

and a curvature relaxation coefficient,

$$
D_{\mathrm{nn}}=\frac{\rho \bar{\kappa}}{L_{\mathrm{nn}}} T
$$

—plus two indirect cross-terms,

$$
D_{\mathrm{dn}}=D_{\mathrm{nd}}=\frac{L_{\mathrm{dn}} \bar{\kappa}}{T},
$$

that represent molecular diffusion induced by curvature and its reciprocal effect of curvature induced by molecular diffusion, respectively.

\section{DISCUSSION}

In summary, Eqs. (25) describe the relaxation of a twocomponent (amphiphilic and nonamphiphilic) fluid membrane towards equilibrium at constant temperature and constant lateral tension. The result was obtained by combining nematohydrodynamics and geometry under the important assumption (18), which is equivalent to the statement that

$$
\frac{\partial}{\partial t}\left(g^{\alpha \beta} \boldsymbol{r}_{\alpha}\right)=g^{\alpha \beta} \boldsymbol{r}_{\alpha} \cdot\left(\nabla \boldsymbol{v}_{l}\right)
$$

That is, the explicit time dependence of the tangent vectors (and their weights) is fixed to be a function of the velocity gradients. Or, more heuristically, since the lipids (amphiphiles) are constrained to point normal to the surface, there is no way of deforming the membrane without moving the relative positions of the amphiphiles and nonamphiphiles. The result predicts that a membrane with a curvature that has nonzero Laplacian-e.g., a parabolic profile-will relax towards a constant-curvature surface accompanied by a simultaneous flow of lipids away from regions where the Laplacian of the curvature is largest (see Fig. 1). Indeed, the equations also predict the inverse effect: If a concentration gradient (with nonzero divergence) can be established in a free membrane, then it would induce a corresponding curvature. An intuitive understanding of this effect can be gained by thinking in terms of molecular splay: the degree to which the head groups of the lipids are separated with respect to their tails. In regions of high curvature, the splay-and hence the energy associated with the configuration - is very high. However, since the molecules are fluid, they can address this situation by displacing nonamphiphilic molecules to increase their concentrationand reduce their splay-at points where the gradient of the curvature has nonzero divergence. Similarly, an enforced (zero divergence) concentration gradient of lipids is not optimal on a constant-curvature surface due to the repulsive effect of too little splay [recall the quadratic profile (4)] and hence the surface-free to move-adjusts its conformation accordingly.

We remark that the form of diffusion coefficients (26) to (28) acts reinforce this picture. For example, if the effective bending modulus $\bar{\kappa}$ is increased-i.e., by using different 

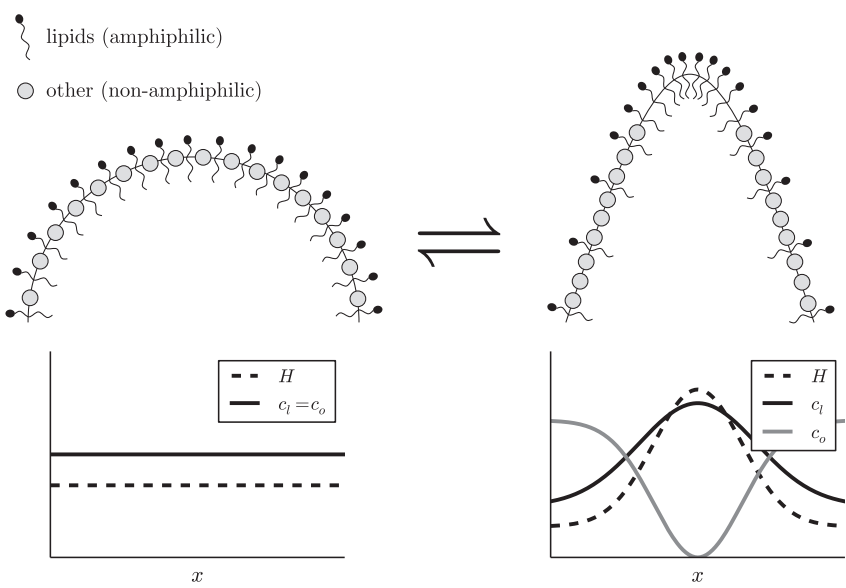

FIG. 1. Exaggerated cartoon indicating the behavior described by Eqs. (25). At constant curvature, the concentrations of both amphiphilic and nonamphiphilic components are spatially uniform. Bending the membrane so that the curvature has a gradient with non-zero divergence (e.g., parabolic, as shown) induces a flow of amphiphilic molecules towards the region where $\nabla^{2} H$ is largest (i.e., the tip), displacing any nonamphiphilic molecules. The reverse process is also allowed: Starting with a parabolic membrane and nonuniform concentrations, the system relaxes to a state of both spatially uniform curvature and molecular concentrations.

lipids - then the rate of the cross diffusion is also increased. That is, if the membrane is stiffer, and more energy has to be added to the system to induce, say, a parabola (as in Fig. 1), then the rate at which lipids flow to reduce the energy of the system is increased by the same factor.

Such effects should, in principle, be observable by experiments that monitor single particle diffusion [30] on a pseudo-free membrane (very large vesicle or sheet with zero hydrostatic pressure difference), where numerical estimates for the diffusion constants, and hence Onsager coefficients would be welcome. Indeed, the broader theory predicts other cross effects, notably in the presence of a temperature gradient, although this is considered out of the scope of this study.

Finally, while there are undoubtedly drawbacks and limitations inherent with such an approach, it is hoped that the work presented here can be adopted and extended in order to overcome any difficulties. In particular, a better understanding of the types (and behavior) of nonamphiphilic molecule most prevalent in biological membranes would be helpful. Armed with such information, it is plausible that this kind of model could shed light on the role of biological membranes in interesting but unexplained phenomena, such as cell locomotion. We therefore welcome further work in the area.

\section{ACKNOWLEDGMENTS}

R.G.M. thanks the grant CEA/DSM-Energie for financial support when working at the Institut de Physique Théorique (CEA). Similarly, R.G.M. acknowledges the current EPSRC Grant No. EP/E501311/1 and also thanks A. J. McKane of the University of Manchester for providing the initial interest in the subject along with many helpful comments and suggestions at the outset.

\section{APPENDIX A: ELASTIC DESCRIPTION OF A MONOLAYER}

In order to better understand the nematic contributions from the specific Gibbs energy, one may adopt an elastic theory of monolayers attributed to Ref. [9] but also described in Ref. [24]. Retaining the spirit of the original work, we present a variant of this approach and demonstrate how it should be interpreted within the context of LNET. Central to the approach is the asymmetric nature of amphiphilic molecules. The principal idea is that, when close to equilibrium, the free energy of a monolayer can be modeled as two elastic sheets with different elastic moduli. The two elastic sheets approximate the different chemical interactions between the head groups and between the tail groups, of amphiphilic molecules. As described in the main text, it is assumed that $g^{\text {nem }}$ is a sum of two quadratic terms, repeated here for convenience:

$$
g^{\text {nem }}=k_{\mathrm{h}}\left(a_{\mathrm{h}}-a_{\mathrm{h}, 0}\right)^{2}+k_{\mathrm{t}}\left(a_{\mathrm{t}}-a_{\mathrm{t}, 0}\right)^{2} .
$$

Here subscripts are used to indicate either head or tail groups. For example, $a_{\mathrm{h}}=A_{\mathrm{h}} / M$ is the area per unit mass on a surface defined to intersect the head groups of all the molecules, while $a_{\mathrm{t}}=A_{\mathrm{t}} / M$ is defined in a similar way for the molecular tails. Both $k_{\mathrm{h}}$ and $k_{\mathrm{t}}$ are constants, as are the quantities $a_{\mathrm{h}, 0}$ and $a_{\mathrm{t}, 0}$, defined as the area per unit mass at equilibrium for head and tail groups, respectively. The problem with incorporating the above into a two-dimensional thermodynamic description is that Eq. (A1) is technically defined on two separate surfaces. The rest of the Appendix therefore follows the approach described by Evans and Skalak [25] in order to write the Gibbs energy in terms of variables defined on a single common surface.

Consider a sample of monolayer under planar stress, that is, $a_{\mathrm{h}}=a_{\mathrm{t}}=a$. The equilibrium separation of the molecules when confined to a plane is then given by the value of $a$ for which $g$ is a maximum. More formally,

$$
\frac{\partial g^{\text {nem }}}{\partial a}=2 k_{\mathrm{h}}\left(a-a_{\mathrm{h}, 0}\right)+2 k_{\mathrm{t}}\left(a-a_{\mathrm{t}, 0}\right)=0,
$$

which implies

$$
a=\frac{k_{\mathrm{h}} a_{\mathrm{h}, 0}+k_{\mathrm{t}} a_{\mathrm{t}, 0}}{k_{\mathrm{h}}+k_{\mathrm{t}}} \equiv a_{0} .
$$

In order to achieve a planar strain on the monolayer it is necessary to apply an asymmetric stress. For example, if $k_{\mathrm{t}}$ is bigger than $k_{\mathrm{h}}$ then the tension applied in the same plane as the tails will have to be larger than that applied to the heads. Mathematically, the lateral tension applied to the head groups while maintaining planar equilibrium is defined as

$$
\Omega_{\mathrm{h}}=\left.\frac{\partial g^{\mathrm{nem}}}{\partial a_{\mathrm{h}}}\right|_{a_{\mathrm{h}=a_{0}}},
$$

and similarly for $\Omega_{\mathrm{t}}$. It is then possible to define the so-called neutral surface, which exists between the head and tail groups and is uniquely defined as the surface of points for which the moments acting on the molecules in planar equilibrium are zero. Here, the word planar is important, as true equilibrium would obviously lead to a curved monolayer (for $k_{\mathrm{h}} \neq k_{\mathrm{t}}$ ). The 
surface is defined by the equation

$$
\Delta \Omega_{\mathrm{h}} \delta_{\mathrm{h}}+\Delta \Omega_{\mathrm{t}} \delta_{\mathrm{t}}=0,
$$

where $\delta_{\mathrm{h}}$ and $\delta_{\mathrm{t}}$ define the distance from the neutral surface to the head and tail groups, respectively. The quantity $\Delta \Omega_{\mathrm{h}}$ is the small change in lateral tension acting at the head groups which arises from a small change in the planar separation. From the definitions above, it is clear that $\Delta \Omega_{\mathrm{h}}=2 k_{\mathrm{h}} \Delta a$ and similarly for $\Delta \Omega_{\mathrm{t}}$. Substituting into Eq. (A5) it follows that

$$
\frac{\delta_{\mathrm{h}}}{\delta_{\mathrm{t}}}=\frac{k_{\mathrm{t}}}{k_{\mathrm{h}}} \text {. }
$$

From here, it is then possible to find expressions for $a_{\mathrm{h}}$ and $a_{\mathrm{t}}$ in terms of $a$, the area per molecule on the neutral surface, and distances $\delta_{\mathrm{h}}$ and $\delta_{\mathrm{t}}$. Geometrical relations of this type are discussed in detail in [8]; therefore, here it suffices to simply state the results

$$
a_{\mathrm{h}}=a\left[1+\delta_{\mathrm{h}} 2 H+\delta_{\mathrm{h}}^{2} K+\mathcal{O}\left(\delta_{\mathrm{h}}^{3}\right)\right],
$$

and

$$
a_{\mathrm{t}}=a\left[1-\delta_{\mathrm{t}} 2 H+\delta_{\mathrm{t}}^{2} K+\mathcal{O}\left(\delta_{\mathrm{t}}^{3}\right)\right],
$$

where two assumptions have been made: first, that $\delta_{\mathrm{h}}$ and $\delta_{\mathrm{t}}$ are of the same order of magnitude, and, second, that the thickness of the membrane is small on the scale of any reasonable curvature. As such, terms of order greater than $\delta_{\mathrm{h}}^{2}$ and $\delta_{\mathrm{t}}^{2}$ have been neglected. Substituting Eqs. (A7) and (A8) into Eq. (A1) leads to an expression for the free energy in terms $a, H$, and $K$. Using Eq. (A6) and the fact that $\delta=\delta_{\mathrm{h}}+\delta_{\mathrm{t}}$, the resultant expression can be manipulated so that terms of the same order in $\delta$ may be grouped together. This gives the expressions referenced in the main text,

$$
g^{\text {nem }}=k\left(a-a_{0}\right)^{2}+\frac{\kappa}{2}\left(2 H-C_{0}\right)^{2}+\kappa_{g} K,
$$

where

$$
\begin{gathered}
k=k_{\mathrm{h}}+k_{\mathrm{t}}, \\
\kappa=\frac{2 k_{\mathrm{h}} k_{\mathrm{t}} a^{2} \delta^{2}}{k}, \\
C_{0}=\frac{a_{\mathrm{h}, 0}-a_{\mathrm{t}, 0}}{a \delta},
\end{gathered}
$$

and

$$
\kappa_{g}=2 \kappa\left[1-\frac{a_{\mathrm{h}, 0}+a_{\mathrm{t}, 0}-a_{0}}{k a}\right] .
$$

\section{APPENDIX B: GEOMETRY}

The two-dimensional surface (embedded in three dimensions) which represents the membrane is defined by a vector field $\boldsymbol{r}=\boldsymbol{r}(u, v)$, where $u$ and $v$ parametrize the surface. The tangent (vector) space associated with each point on the surface is then spanned by vectors $\boldsymbol{r}_{\alpha} \equiv \partial \boldsymbol{r} / \partial q^{\alpha}$, where $\alpha \in\{1,2\}, q^{1}=u$, and $q^{2}=v$. From here, the first fundamental form, or metric, is defined as

$$
g_{\alpha \beta} \equiv \boldsymbol{r}_{\alpha} \cdot \boldsymbol{r}_{\beta}
$$

where the inverse metric $g^{\alpha \beta}$ is defined such that $g^{\alpha \beta} g_{\beta \gamma}=\delta_{\gamma}^{\alpha}$, with $\delta_{\gamma}^{\alpha}$ the Kronecker $\delta$ symbol. Here, $g$ is the determinant of $g_{\alpha \beta}$, given by

$$
g \equiv \frac{1}{2} \varepsilon^{\alpha \gamma} \varepsilon^{\beta \nu} g_{\alpha \beta} g_{\gamma \nu},
$$

where $\varepsilon^{\alpha \beta}$ is an antisymmetric two-dimensional Levi-Civita symbol. The determinant is used to define the surface area element,

$$
d A \equiv \sqrt{g} d u d v
$$

and the unit normal

$$
\hat{\boldsymbol{n}} \equiv \frac{\boldsymbol{r}_{1} \times \boldsymbol{r}_{2}}{\sqrt{g}},
$$

where, for notational simplicity, the traditional "hat" notation is omitted going forwards. In order to quantify the curvature of a surface it is further necessary to define second derivatives $\boldsymbol{r}_{\alpha \beta} \equiv \partial^{2} \boldsymbol{r} / \partial q^{\alpha} \partial q^{\beta}$, where the coefficients of the second fundamental form,

$$
L_{\alpha \beta} \equiv \boldsymbol{r}_{\alpha \beta} \cdot \boldsymbol{n},
$$

and their determinant,

$$
l \equiv \frac{1}{2} \varepsilon^{\alpha \gamma} \varepsilon^{\beta \nu} L_{\alpha \beta} L_{\gamma \nu},
$$

allow us to make contact with (1) by writing

$$
H \equiv-\frac{1}{2} g^{\alpha \beta} L_{\alpha \beta}
$$

and

$$
K \equiv \frac{l}{g},
$$

where a sum is implicit over repeated indices. For consistency with (1) and the majority of membrane related literature, (B7) is defined here contrary to the usual conventions of differential geometry, so that the mean curvature of a sphere is positive, $H_{\text {sphere }}=1 / R$.

In order to perform the derivatives present in the definition of the molecular field, it is necessary to write the mean and Gaussian curvatures as functions of the unit normal and its spatial derivatives. We start with the mean curvature. Since $\boldsymbol{r}_{\alpha} \cdot \boldsymbol{n}=0$ then $L_{\alpha \beta}=-\boldsymbol{r}_{\alpha} \cdot \boldsymbol{n}_{\beta}$. Substituting into (B7) and rewriting in terms of the gradient operator,

$$
\nabla \equiv g^{\alpha \beta} \boldsymbol{r}_{\alpha} \partial_{\beta},
$$

gives the standard result

$$
H=\frac{1}{2} \nabla \cdot \boldsymbol{n} .
$$

Rewriting the Gaussian curvature is slightly more involved. We use two relations without proof [31]: the Weingarten relation,

$$
\boldsymbol{n}_{\alpha}=-L_{\alpha \beta} g^{\beta \gamma} \boldsymbol{r}_{\gamma}
$$

and the lesser known result,

$$
g^{\alpha \beta} L_{\beta \gamma} g^{\gamma \nu} L_{\nu \alpha}=4 H^{2}-2 K .
$$

Our starting point comes from combining these two equations, where it is straightforward to see that

$$
\left(\boldsymbol{n}_{\alpha} \cdot \boldsymbol{n}_{\beta}\right) g^{\alpha \beta}=4 H^{2}-2 K .
$$

Here the left-hand side can be rewritten by using the fact that $\partial_{\alpha}\left(|\boldsymbol{n}|^{2}\right)=0$ and therefore $\boldsymbol{n} \cdot \boldsymbol{n}_{\alpha}=0$, which implies that 
$\left(\boldsymbol{n}_{\alpha} \cdot \boldsymbol{n}_{\beta}\right) g^{\alpha \beta}=-\boldsymbol{n} \cdot \partial_{\alpha}\left(g^{\alpha \beta} \partial_{\beta} \boldsymbol{n}\right)$. Introducing the LaplaceBeltrami operator,

$$
\nabla^{2} \equiv \nabla \cdot \nabla=\frac{1}{\sqrt{g}} \partial_{\alpha}\left(\sqrt{g} g^{\alpha \beta} \partial_{\beta}\right),
$$

it is relatively simple to show that

$$
\boldsymbol{n} \cdot \nabla^{2} \boldsymbol{n}=\boldsymbol{n} \cdot \partial_{\alpha}\left(g^{\alpha \beta} \partial_{\beta} \boldsymbol{n}\right),
$$

and hence Eq. (B13) can be inverted to show that

$$
K=\frac{1}{2}\left[(\nabla \cdot \boldsymbol{n})^{2}+\boldsymbol{n} \cdot\left(\nabla^{2} \boldsymbol{n}\right)\right] .
$$

\section{APPENDIX C: APPLICATION OF THERMODYNAMIC CONSERVATION LAWS}

As described in the main text, the local Gibbs relation can be transformed by applying thermodynamic conservation laws. Conservation laws of this type are commonplace in fluid dynamics and therefore simply stated here, with the full physical justification provided elsewhere (see, e.g., [23]). We start with local conservation of mass which is unchanged by the inclusion of nematic media, giving

$$
\frac{d c_{k}}{d t}=-\frac{1}{\rho} \nabla \cdot J_{k},
$$

where

$$
\boldsymbol{J}_{k}=\rho_{k}\left(\boldsymbol{v}_{k}-\boldsymbol{v}\right),
$$

is the local diffusion flow. Here $\rho_{k} \equiv M_{k} / V$ are the partial mass densities, while the relation $\boldsymbol{v}=\sum_{k} \boldsymbol{v}_{k} \rho_{k} / \rho$ defines both the barycentric (or center-of-mass) velocity $\boldsymbol{v}$, and the partial velocities $\boldsymbol{v}_{k}$. In a similar way, the conservation of local heat $q$ is also unchanged by nematic effects, with the standard relation given by

$$
\frac{d q}{d t}=-\frac{1}{\rho} \nabla \cdot J_{\mathrm{q}},
$$

where $\boldsymbol{J}_{\mathrm{q}}$ is the local heat flow. Before writing down the conservation of internal energy, however, note that a general analysis of the anisotropic term $\{d g\}_{T, p}, c_{k}$ leads to a consideration of friction that would not otherwise be present for a simple fluid. As a result, it is necessary to briefly review the general equation of motion for a fluid, given by

$$
\rho \frac{d \boldsymbol{v}}{d t}=-\nabla \cdot \mathrm{P},
$$

where $P$ is the stress tensor. Assuming that the constituents of the fluid are inelastic it is natural to decompose the pressure tensor into a hydrostatic part $p$, and a tensor $\Pi$, such that

$$
\mathrm{P}=p \mathbf{I}+\Pi,
$$

where I is the identity matrix. In general, if the constituent particles are anisotropic, $\Pi$ and therefore $P$ are not symmetric. Taking this into account, local conservation of internal energy becomes

$$
\frac{d u}{d t}=\frac{d q}{d t}-\frac{1}{\rho} \Pi:(\nabla v)^{\top}-\frac{d \nu}{d t},
$$

where the notation is described in the main text. Using this result alongside conservation of heat and conservation of
mass-Eqs. (C3) and (C1), respectively_it follows that

$$
\begin{aligned}
\rho T \frac{d s}{d t}= & -\nabla \cdot \boldsymbol{J}_{\mathrm{q}}-\Pi:(\nabla \boldsymbol{v})^{\top} \\
& +\sum_{k} \mu_{k} \nabla \cdot \boldsymbol{J}_{k}-\rho\left\{\frac{d g}{d t}\right\}_{T, p, c_{k}},
\end{aligned}
$$

which is exactly the Eq. (10) from the main text if the identifications (6), also in the main text, are made.

\section{APPENDIX D: CONSERVATION OF ANGULAR MOMENTUM}

The main idea of this Appendix is to temporarily imagine that the lipid molecules were not fixed to point normal to the membrane and then implement conservation of angular momentum by following [29]. Once conservation angular momentum has been imposed, it is then easier to ascertain the impact of enforcing the "Helfrich condition": that the director is always normal to a surface. (Since the material contained in Ref. [29] is presented there in a disparate way across a number of chapters, this Appendix provides a systematic, if terse, formulation which has the benefit of being notationally consistent with the main text.)

First, it is necessary to recognize that the free energy of a nematic is unchanged if the both the molecular positions and the director are rotated by small amount. More formally, consider the following deformations to position vector $\boldsymbol{r}$ and director $\boldsymbol{n}$, respectively:

$$
\delta \boldsymbol{r}=\boldsymbol{\omega} \times \boldsymbol{r} \quad \text { and } \quad \delta \boldsymbol{n}=\boldsymbol{\omega} \times \boldsymbol{n} .
$$

In component form, these relations become

$$
\nabla_{i}(\delta r)_{j}=\varepsilon_{i j l} \omega_{l} \quad \text { and } \quad(\delta n)_{i}=\varepsilon_{i j l} \omega_{j} n_{l},
$$

where indices $i, j$, and $l$ label Cartesian components ( $k$ is reserved for labeling the components of the mixture) and $\varepsilon_{i j l}$ is the totally antisymmetric Levi-Civita symbol. It can be seen that the total variation in the Gibbs energy due to small deformations is given by

$$
\rho \delta g=-\Pi_{i j}^{d} \nabla_{i}(\delta r)_{j}+h_{i}(\delta n)_{i}+\nabla_{i}\left[\rho \frac{\partial g}{\partial\left(\nabla_{i} n_{j}\right)}(\delta n)_{j}\right] .
$$

Substituting Eqs. (D2) into the above and setting $\delta g=0$ gives $0=-\Pi_{i j}^{d} \varepsilon_{i j l} \omega_{l}+h_{i} \varepsilon_{i j l} \omega_{j} n_{l}+\nabla_{i}\left[\rho \frac{\partial g}{\partial\left(\nabla_{i} n_{j}\right)} \varepsilon_{j l m} \omega_{l} n_{m}\right]$,

where, by relabeling the indices of the second term, a common factor of (constant vector) $\omega_{l}$ may be removed. Integrating this result over the entire volume, and using the divergence theorem [32], leads to the relation

$$
\begin{aligned}
0= & -\int \Pi_{i j}^{d} \varepsilon_{i j l} d V+\int \varepsilon_{i j l} n_{i} h_{j} d V \\
& +\int d A_{i}\left[\rho \frac{\partial g}{\partial\left(\nabla_{i} n_{j}\right)}\right] \varepsilon_{m j l} n_{m},
\end{aligned}
$$

where $d A_{i}$ is the $i$ th component of the surface area element $\boldsymbol{d A}$. With this relation in mind, it is necessary to temporarily 
turn attention to the nematic nature of the molecules. Due to their rodlike form, a sample of nematic material must obey conservation of angular momentum. The rate of change of total angular momentum is given by

$$
\frac{d L}{d t}=\frac{d}{d t} \int \rho(\boldsymbol{r} \times \boldsymbol{v}) d V
$$

The time derivative may be taken inside this integral by using Liebniz's rule,

$$
\frac{d L}{d t}=\int \frac{\partial}{\partial t}[\rho(\boldsymbol{r} \times \boldsymbol{v})] d V+\int \rho(\boldsymbol{r} \times \boldsymbol{v}) \boldsymbol{v} \cdot d \boldsymbol{A} .
$$

Manipulating derivatives and using conservation of mass it follows that

$$
\frac{d L}{d t}=\int \rho \frac{d}{d t}(\boldsymbol{r} \times \boldsymbol{v}) d V=-\int \boldsymbol{r} \times(\nabla \cdot \mathrm{P}) d V .
$$

Here the final step comes from the equation of motion (C4). The resultant expression may then be integrated by parts. In component form this gives

$$
\frac{d L}{d t}=-\int \varepsilon_{i j l} r_{j} P_{m l} d A_{m}+\int \varepsilon_{i j l} P_{j l} d V,
$$

where the second term on the right-hand side can be simplified further by splitting the pressure tensor into hydrostatic and tensor parts, $P_{i j}=\Pi_{i j}+p \delta_{i j}$; cf. Eq. (C5). The result is that

$$
\frac{d L}{d t}=-\int \varepsilon_{i j l} r_{j} P_{m l} d A_{m}+\int \varepsilon_{i j l} \Pi_{j l} d V .
$$

However, following de Gennes [29], the rate of change of angular momentum is also equal to the total torque due to external stresses acting at the boundary, given by

$$
-\int \boldsymbol{r} \times(\mathrm{P} \cdot d \boldsymbol{A}),
$$

plus the total torque on the director at the boundary

$$
\int \boldsymbol{n} \times\left[\rho \frac{\partial g}{\partial(\nabla \boldsymbol{n})} \cdot d \boldsymbol{A}\right] .
$$

In component form, this can be written as

$$
\frac{d L}{d t}=\int d A_{m} \varepsilon_{i j l}\left\{n_{j}\left[\rho \frac{\partial g}{\partial\left(\nabla_{m} n_{l}\right)}\right]-r_{j} P_{m l}\right\} .
$$

Comparison with Eq. (D10) leads to the result

$$
\int d V \varepsilon_{i j l} \Pi_{j l}=\int d A_{m} \varepsilon_{i j l} n_{j}\left[\rho \frac{\partial g}{\partial\left(\nabla_{m} n_{l}\right)}\right] .
$$

Finally, this may be combined with Eq. (D5) to eliminate the surface integral on the right-hand side. The result is that

$$
\varepsilon_{i j l} \Pi_{j l}^{\prime}=-\varepsilon_{i j l} n_{j} h_{l}
$$

In order to understand this, it is useful to consider decomposing the viscous stress tensor into symmetric and antisymmetric parts. Introducing superscripts $s$ and a to denote symmetric and antisymmetric, respectively, gives

$$
\Pi_{i j}^{\prime}=\Pi_{i j}^{\mathrm{a}}+\Pi_{i j}^{\mathrm{s}}
$$

where the elements of the antisymmetric part are defined in the following way:

$$
\begin{aligned}
& \Pi_{12}^{\mathrm{a}}=-\Pi_{21}^{\mathrm{a}}=\frac{1}{2} \varepsilon_{3 j l} \Pi_{j l}^{\prime}, \\
& \Pi_{23}^{\mathrm{a}}=-\Pi_{32}^{\mathrm{a}}=\frac{1}{2} \varepsilon_{1 j l} \Pi_{j l}^{\prime}, \\
& \Pi_{31}^{\mathrm{a}}=-\Pi_{13}^{\mathrm{a}}=\frac{1}{2} \varepsilon_{2 j l} \Pi_{j l}^{\prime} .
\end{aligned}
$$

The viscous stress tensor arises in the expression for entropy production in an inner product with the transpose of the velocity gradient tensor, $(\nabla \boldsymbol{v})^{\top}$. Indeed, it is also possible to split the velocity gradient tensor into symmetric and antisymmetric parts. In this way, the dyadic product splits into two separate dyadics between symmetric and antisymmetric parts,

$$
\Pi:(\nabla v)^{\top}=\Pi^{\mathrm{s}}:(\nabla \boldsymbol{v})^{\mathrm{s}}+\Pi^{\mathrm{a}}:(\nabla \boldsymbol{v})^{\mathrm{T}, \mathrm{a}},
$$

where the notation $(\cdots)^{\mathrm{T}}$, a indicates the antisymmetric part of the transpose (which is equal to the transpose of the antisymmetric part). This can be expressed more easily in component form

$$
\Pi_{i j} \nabla_{i} v_{j}=\Pi_{i j}^{\mathrm{s}}\left(\nabla_{i} v_{j}\right)^{\mathrm{s}}+\Pi_{i j}^{\mathrm{a}}\left(\nabla_{i} v_{j}\right)^{\mathrm{a}}
$$

where repeated indices imply a sum. Here, as with Eqs. (D17), the three independent parts of the antisymmetric velocity gradient tensor may be linked to the components of the vector,

$$
\omega_{i}=\frac{1}{2} \varepsilon_{i j k} \nabla_{j} v_{k}
$$

Combining this with Eqs. (D17), the dyadic between antisymmetric parts which arises in Eq. (D19) may be rewritten as

$$
\Pi_{i j}^{\mathrm{a}}\left(\nabla_{i} v_{j}\right)^{\mathrm{a}}=-\frac{1}{4} \varepsilon_{i j l} \varepsilon_{i m n} \Pi_{j l}^{\prime} \nabla_{m} v_{n}
$$

Here it is possible to invoke Eq. (D15), the result of both symmetry considerations and angular momentum conservation. It follows that

$$
\Pi^{\mathrm{a}}:(\boldsymbol{\nabla} \boldsymbol{v})^{\mathrm{T}, \mathrm{a}}=\frac{1}{2} \boldsymbol{\omega} \cdot(\boldsymbol{n} \times \boldsymbol{h}),
$$

where $\omega=(\nabla \times v) / 2$ is recognized as the vorticity. Using the properties of the scalar triple product, it it clear that

$$
\Pi^{\prime}: \nabla v+\boldsymbol{h} \cdot \dot{\boldsymbol{n}}=\Pi^{\mathrm{s}}:(\nabla \boldsymbol{v})^{\mathrm{s}}+\boldsymbol{h} \cdot \boldsymbol{N},
$$

where $\boldsymbol{N}=\dot{\boldsymbol{n}}-(\boldsymbol{\omega} \times \boldsymbol{n}) / 2$ is the rate of change of the director relative to the background fluid.

At this stage we simply apply the Helfrich condition by assuming that the director corresponds to the normal of a regular two-dimensional surface, embedded in three dimensions. Immediately, one can see that $\Pi^{\mathrm{a}}:(\boldsymbol{\nabla} \boldsymbol{v})^{\mathrm{T}}$, a $=0$ and therefore $N=\dot{\boldsymbol{n}}$. The result is that

$$
\Pi^{\prime}: \nabla \boldsymbol{v}+\boldsymbol{h} \cdot \dot{\boldsymbol{n}}=\Pi^{\mathrm{s}}:(\nabla \boldsymbol{v})^{\mathrm{s}}+\boldsymbol{h} \cdot \dot{\boldsymbol{n}}
$$

which is used in the result (15) of the main text. 
[1] B. Alberts et al., Molecular Biology of the Cell (Garland Science, New York, 1989).

[2] D. D. Lasic, in Structure and Dynamics of Membranes-From Cells to Vesicles, edited by R. Lipowsky and E. Sackmann, Handbook of Biological Physics Vol. 1, Part 1 (North-Holland, Amsterdam, 1995), pp. 491-519.

[3] U. Seifert, Adv. Phys. 46, 13 (1997).

[4] W. Helfrich, Z. Naturforsch. 28c, 693 (1973).

[5] P. B. Canham, J. Theor. Biol. 26, 61 (1970).

[6] E. A. Evans, Biophys. J. 14, 923 (1974).

[7] F. C. Frank, Discuss. Faraday Soc. 25, 19 (1958).

[8] D. C. Kay, Schaum's Outline of Theory and Problems of Tensor Calculus (McGraw-Hill, New York, 1998).

[9] S. Svetina, M. Brumen, and B. Žekš, Stud. Biophys. 110, 177 (1985).

[10] L. Miao, U. Seifert, M. Wortis, and H.-G. Döbereiner, Phys. Rev. E 49, 5389 (1994).

[11] H. J. Deuling and W. Helfrich, J. Phys. 37, 1335 (1976).

[12] J. T. Jenkins, J. Math. Biol. 4, 149 (1977).

[13] J. C. Luke and J. I. Kaplan, Biophys. J. 25, 107 (1979).

[14] J. C. Luke, SIAM J. Appl. Math. 42, 333 (1982).

[15] M. A. Peterson, J. Appl. Phys. 57, 1739 (1985).

[16] U. Seifert, K. Berndl, and R. Lipowsky, Phys. Rev. A 44, 1182 (1991).

[17] H.-G. Döbereiner, E. Evans, M. Kraus, U. Seifert, and M. Wortis, Phys. Rev. E 55, 4458 (1997).
[18] D. Fanelli and A. J. McKane, Phys. Rev. E 78, 051406 (2008).

[19] B. Božič and S. Svetina, Phys. Rev. E 80, 013401 (2009).

[20] D. Fanelli and A. J. McKane, Phys. Rev. E 80, 013402 (2009).

[21] R. G. Morris, D. Fanelli, and A. J. McKane, Phys. Rev. E 82, 031125 (2010).

[22] R. G. Morris and A. J. McKane, Phys. Rev. E 83, 061151 (2011).

[23] S. R. de Groot and P. Mazur, Non-equilibrium Thermodynamics (Dover Publications, New York, 1984).

[24] A. G. Petrov and I. Bivas, Prog. Surf. Sci. 16, 389 (1984).

[25] E. A. Evans and R. Skalak, Mechanics and Thermodynamics of Biomembranes (CRC Press, Boca Raton, Florida, 1980), No. 4.

[26] J. L. Ericksen, J. Rheol. 5, 23 (1961).

[27] F. M. Leslie, Arch. Ration. Mech. Anal. 28, 265 (1968).

[28] N. Ogawa, Phys. Rev. E 81, 061113 (2010).

[29] P. G. de Gennes, The Physics of Liquid Crystals (Oxford University Press, Oxford, 1974), Chap. 5.

[30] G. Gopalakrishnan et al., Angew. Chem. (International edition in English) 45, 5478 (2006).

[31] Z.-C. Ou-Yang, J.-X. Liu, and Y.-Z. Xie, Geometric Methods in the Elastic Theory of Membranes in Liquid Crystal Phases (World Scientific, Singapore, 1999).

[32] G. B. Arfken, Mathematical Methods for Physicists (Elsevier, Amsterdam, 1985). 